\title{
Laparoscopic Removal of a Broken Sewing Needle in a Patient with Irritative Bladder Symptoms
}

\author{
Jun Taik Lee, Hong Koo Ha ${ }^{1}$, Dong Gil Shin ${ }^{1}$, Wan Lee ${ }^{1}$, Zeong Zoo Lee ${ }^{1}$ \\ Department of Urology, Busan St. Mary’s Medical Center, Busan; \\ ${ }^{1}$ Department of Urology, Pusan National University Hospital, Pusan National University School of Medicine, Busan, Korea
}

\begin{abstract}
A foreign body near the bladder is rare. Although foreign bodies in the bladder can be easily found and removed by endoscopic transurethral removal, extravesical foreign bodies may require the use of an open or laparoscopic procedure. Here, we report a case of a patient complaining of frequency and urgency in whom an extravesical sewing needle was successfully removed by a laparoscopic approach. A $4.5 \mathrm{~cm}$ rusty sewing needle was found between the bladder and the left external iliac vessels and was removed through a $5 \mathrm{~mm}$ trocar port by use of endo forceps with no complications.
\end{abstract}

Keywords: Foreign bodies; Urinary bladder; Laparoscopy

The accidental identification of foreign bodies in human tissue is rather frequent, especially in the case of sewing needles. In general, such objects can cause discomfort or can become lodged near an organ causing a risk of perforation, often necessitating surgical removal [1]. There are some case reports of the intravesical migration of intrauterine devices or retained surgical foreign bodies: however, a case of a sewing needle in the pelvis has rarely been reported. Here, we report the laparoscopic removal of a sewing needle from the pelvis of a patient with irritative bladder symptoms.

\section{CASE REPORT}

An 84-year-old man was transferred to our institute because of a foreign object observed in the perivesical space on radiographic examination. He had dementia, hypertension, and bilateral pleural effusion. He had not undergone prior surgery. Because of his cognitive and memory dysfunctions, he could not remember exactly how the foreign material had entered his body; however, he suggested that he sat on a cushion on which there was a needle several days ago, and he complained of urinary symp- toms including frequency and urgency. No significant skin injury around the back, buttocks, or perineal region was found in the physical examination. On the transrectal ultrasonogram, the prostate and adenoma volumes were $48 \mathrm{~mL}$ and $23 \mathrm{~mL}$, respectively.

A sharp needle-like material was visible in the pelvic cavity on plain pelvic images (Fig. 1). Non-enhanced computed tomography showed a $4.5 \mathrm{~cm}$ broken, needle-like material at the right lateral bladder wall (Fig. 2). We found no abnormalities except for bladder trabeculation and inflammatory change by cystourethroscopy. There were no specific findings except elevated C-reactive protein $(2.84 \mathrm{mg} / \mathrm{dL})$ on the laboratory examinations.

A fan-shaped three-port laparoscopic extraperitoneal approach was performed (one 10-mm port placed at the umbilicus [camera], two $5 \mathrm{~mm}$ working ports placed at each side). Mild adhesion was identified around the perivesical space. While dissecting the bladder and the prostate, I could feel an abnormal material on the right side of the bladder. A needle adhesive to adjacent tissues was encountered between the right side of the bladder and the right external iliac vessels (Fig. 3). The two parts
Corresponding author: Hong Koo Ha

Department of Urology, Pusan National University Hospital, Pusan National University School of Medicine, 179 Gudeok-ro, Seo-gu, Busan 602-739, Korea Tel: +82-51-240-7351 / Fax: +82-51-247-5443 / E-mail: hongkooha@pusan.ac.kr Submitted: November 22, 2010 / Accepted after revision: December 9, 2010
This is an Open Access article distributed under the terms of the Creative Commons Attribution Non-Commercial License (http://creativecommons.org/licenses/by-nc/3.0/) which permits unrestricted non-commercial use, distribution, and reproduction in any medium, provided the original work is properly cited. 


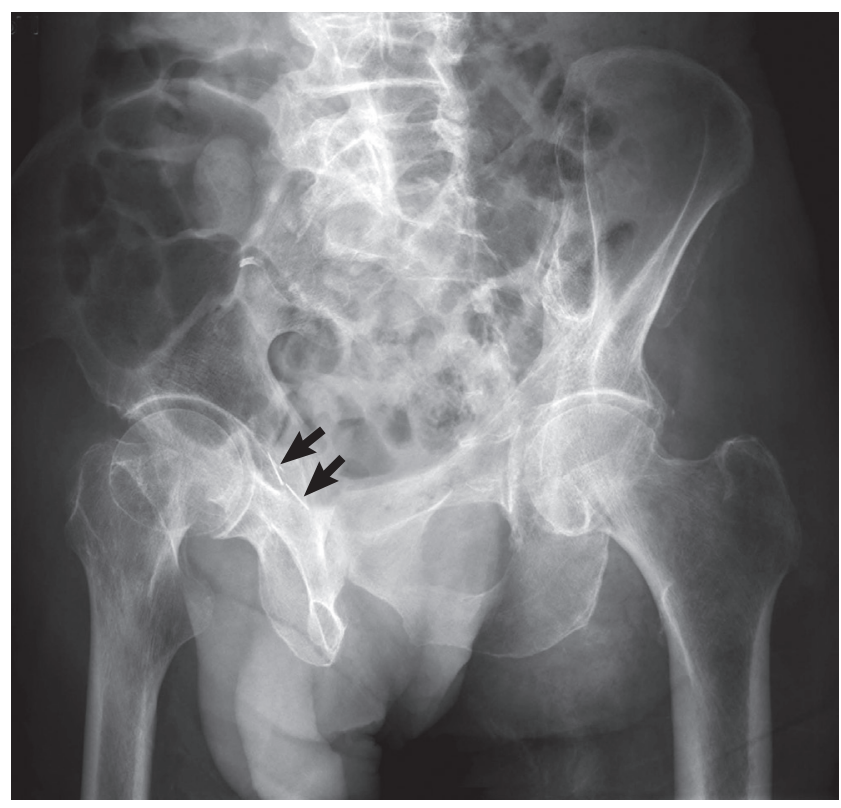

Fig. 1. A sewing-needle-like foreign body (arrows) is visible just above the right pubic ramus on the plain oblique pelvic image.

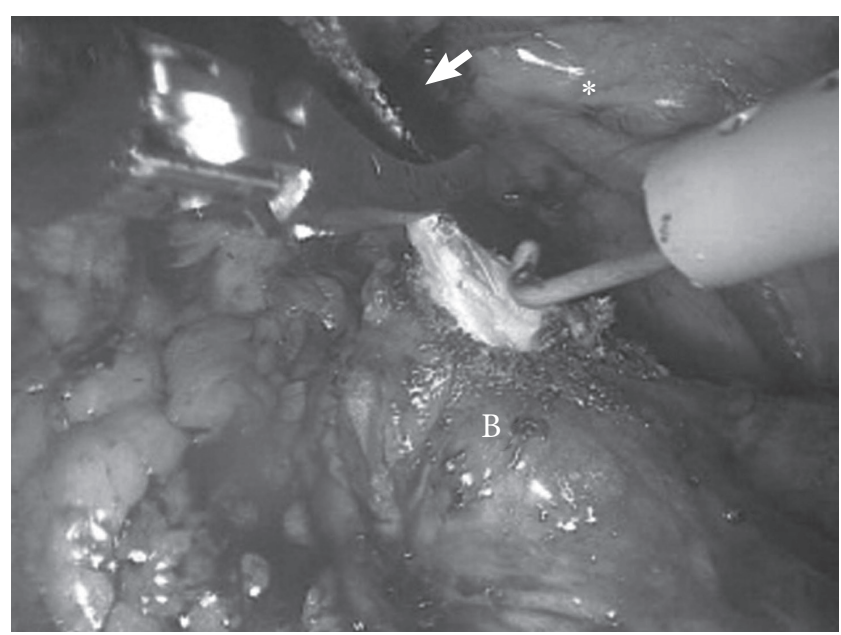

Fig. 3. Intraoperative findings. A sewing needle (arrow) was adhered to adjacent tissues between the bladder and the right external iliac vessels. The operator is dissecting the sewing needle from detrusor muscle fibers. B, bladder; *, right pubic ramus.

of the needle were identified and were removed carefully through the laparoscopic port (Fig. 4).

The operation took 45 minutes. The estimated bleeding was $50 \mathrm{~mL}$, and no transfusion was needed. The pain could be controlled by administration of oral analgesics, and the patient could walk by the first postoperative day. Even though the patient's voiding symptoms were moderately improved after the surgical removal of the needle, we prescribed an a-blocker because of

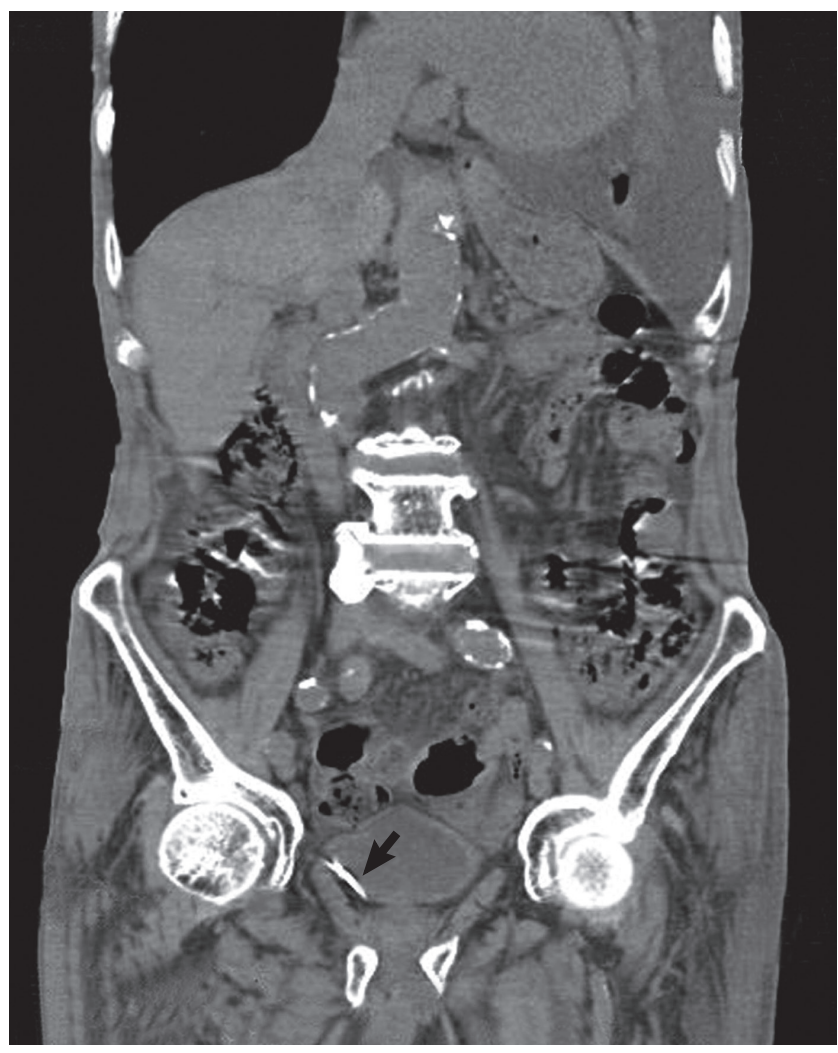

Fig. 2. The non-enhanced computed tomography image of the pelvis shows a foreign body with a high signal intensity on the right side of the bladder (arrow).

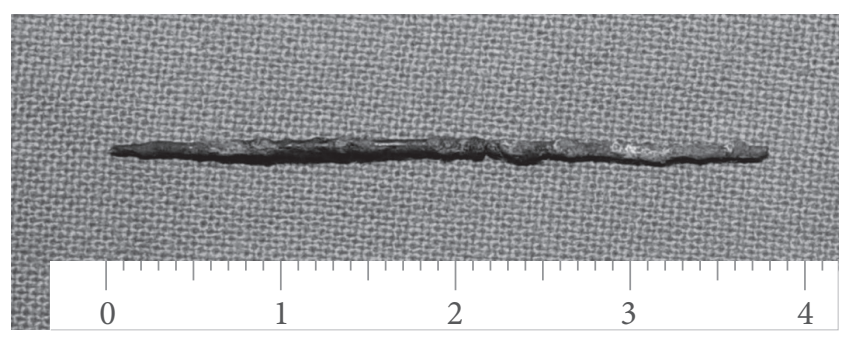

Fig. 4. The broken sewing needle.

the large prostate. The International Prostate Symptom Score (IPSS) decreased from 32 preoperatively to 13 postoperatively. Especially, urge urinary symptom score at IPSS was decreased from 5 to 1 point and frequency was from 4 to 2 . However, nocturia and weak urinary stream were not improved after operation. These symptoms improved after administration of an a-blocker.

\section{DISCUSSION}

In a review of the literature, I found a few reports of a sewing 
needle in the intraperitoneal space that was introduced iatrogenically or by self-ingestion [2], and only one case in which a sewing needle was reported in the extraperitoneum [3]. Only two case reports have presented the surgical removal of a needle from the extravesical space. Kim et al. [3] reported the laparoscopic removal of a rusty needle around the vesicouterine fold in a woman who had not undergone a prior operation. The patient complained of vaginal discharge, which suggested that the needle had entered her body through the vagina. Izumi et al. [4] reported a bladder stone secondary to migration of an acupuncture needle that was retained in the patient's back 20 years previously.

Foreign bodies in the intraperitoneal or extraperitoneal space result in local irritation symptoms or local infection. In a previous case, an encrusted needle caused pyelonephrosis and sepsis in a patient with a foreign body that passed per orally into the renal pelvis after perforation of the duodenum [5]. Patients with intravesical foreign bodies experience chronic pelvic pain and irritative urinary symptoms [6]. In this case, we could not identify any abnormalities on the patient's body surface during the physical examination; however, the patient complained of frequency and urge urinary symptoms. The needle might have acted as an irritative factor during the filling phase in the bladder. After the surgical removal of the needle, the patient's voiding symptoms, including frequency and urgency, improved slightly. Because he had a large prostate, however, we additionally managed the patient with an alpha-adrenergic blocker. His voiding symptom score was significantly improved after the surgical removal of the needle and administration of an alphablocker.

Radiologic localization of foreign bodies is mandatory when an operation is being considered. Plain abdominal film and non-enhanced computed tomography provide excellent results for the localization of the needle. Nevertheless, it is difficult to localize exactly where the needles are intraoperatively. In the current case, we could not identify on the radiologic images whether the needle had penetrated the bladder, and the cystoscopic findings showed that it was located extravesically.

Improvements in laparoscopic instruments and surgical techniques have resulted in the increased use of the laparoscopic approach, which enables good cosmetic results, postoperative recovery, and, especially, tactile sensation [7]. In this case, despite partial tactile sensation, the laparoscopic procedure was good for localization of the foreign body. Laparoscopic removal of foreign bodies is desirable because it can shorten the duration of postoperative recovery, decrease morbidity, and reduce the incidence of postoperative complications. Jung et al. [7] reported that intravesical foreign bodies can be removed safely and efficiently by use of the laparoscopic approach. In this report, we have described the successful laparoscopic extraction of a broken sewing needle in the pelvis with no complications.

\section{CONFLICT OF INTEREST}

No potential conflict of interest relevant to this article was reported.

\section{REFERENCES}

1. Costa Monteiro E, Hall Barbosa C, Andrade Lima E, Costa Ribeiro P, Boechat P. Locating steel needles in the human body using a SQUID magnetometer. Phys Med Biol 2000;45:2389-402.

2. Ma CJ, Jan CM, Hsieh JS, Huang YS, Wang WM, Chan HM, et al. Successful localization and surgical removal of ingested sewing needles under mini C-arm fluoroscopy: a case report. Kaohsiung J Med Sci 2006;22:457-60.

3. Kim BS, Chong GO, Hong DG, Park NY, Lee YS. Laparoscopic removal of a rusty sewing needle from the pelvis. J Minim Invasive Gynecol 2008;15:129.

4. Izumi K, Takizawa A, Udagawa K, Murai T, Murai M. Bladder stone secondary to migration of an acupuncture needle. Hinyokika Kiyo 2008;54:365-7.

5. Frang D, Rózsahegyi G, Czvalinga I. A swallowed needle found in the kidney pelvis. Z Urol Nephrol 1978;71:647-51.

6. Yensel U, Bezircioglu I, Yavuzcan A, Baloglu A, Cetinkaya B. Migration of an intrauterine device into the bladder: a rare case. Arch Gynecol Obstet 2009;279:739-42.

7. Jung US, Lee JH, Kyung MS, Kim KH, Choi JS. Laparoscopic removal of an intravesical foreign body after laparoscopically assisted vaginal hysterectomy: a case report and review of the literatures. Surg Laparosc Endosc Percutan Tech 2008;18:420-2. 